\title{
“以抛物线为特殊积分的二次系统 的极限环”一文的注记
}

\author{
沈伯寒 \\ 宋燕 \\ （辽宁师范大学, 大连 116022）（锦州师范专科学校, 锦州 121000)
}

\section{关链词 拋物线解、极限环、稳定焦点}

文献 [1]研究了二次系统具有抛物线解时极限环的存在性问题, 把所有有可能存在极限环 时的此类系统分为以下两种形式 (本文均按照原文记号), 即

$$
\left\{\begin{array}{l}
\dot{x}=x y+\mu=P(x, y), \\
\dot{y}=(x y+\mu)(x+l)+(2 y-1)\left(y-\frac{1}{2} x^{2}-l x-m\right)=Q(x, y),
\end{array} \quad(\mu>0)\right.
$$

和

$$
\left\{\begin{array}{l}
\dot{x}=x y+\mu, \\
\dot{y}=(x y+\mu)(x+l)+(2 y+1)\left(y-\frac{1}{2} x^{2}-l x-m\right), \quad(\mu>0)
\end{array}\right.
$$

其中抛物线解的方程为

$$
G(x, y) \equiv y-\frac{1}{2} x^{2}-l x-m=0,
$$

并根据(2)式上奇点的横坐标满足方程

$$
K(x) \equiv x^{3}+2 l x^{2}+2 m x+2 \mu=0
$$

的根的情况, 将系统 (1) 给出了分为 (A)、(B)、(C)、(D)四种情形, 存在极限环的充要条件, 其中 均有 “ $M_{1}$ 为系统 (1) 在 (2)式内侧的稳定焦点”这样一个在具体验证时是不方便的条件. 本文 将用系数间的关系式给出系统 (1) 在各种情况下极限环存在的充要条件, 并指出在情形 (C) 中,当 $a>b$ 时不存在极限环.

定理 1 对情形(B), 系统(1) 存在极限环的充要条件是以下三条件同时被满足: $0<q<1$; (ii) $\lambda<0$; (iii) $\lambda^{2}-8 \lambda+8\left(a^{2} q^{2}-a p-q+2\right)<0$, 其中 $\lambda=a^{2} q+a p q-$ $2 a p-2 q+3$.

证 由文献[1], 与 “ $M_{1}$ 为系统(1)的稳定焦点”等价的条件是以下的 (i)、(ii)、(iii) 同 时成立:

(i) $M_{1}$ 在 $G(x, y)=0$ 的内部, $\Leftrightarrow \frac{1}{2}-2 \mu^{2}+2 \mu l-m>0, \Leftrightarrow a^{2} q^{2}-a^{2} q-$ $a p q+a p+q-1<0, \Leftrightarrow(q-1)\left(a^{2} q-a p+1\right)<0, \Leftrightarrow 0<q<1\left(0 \leqslant p^{2}<4 q\right)$,

本文 1989 年 9 月 4 日收到. 
可祖 $a^{2} q-a p H>0$;

(ii) $M_{1}$ 为稳定的, $\Leftrightarrow 2 \mu l-2 m+\frac{3}{2}<0, \Leftrightarrow a^{2} q+a p q-2 a p-2 q+3<$ 0 ;

(iii) $M_{1}$ 为焦点, $\Leftrightarrow 4 \mu^{2} l^{2}+4 m^{2}+\frac{1}{4}-8 \mu l m-2 \mu l-2 m+8 \mu^{2}<0, \Leftrightarrow a^{4} q^{2}$ $+2 a^{3} p q^{2}+a^{2} p^{2} q^{2}+4 a^{2} p^{2}+6 a p q+4 q^{2}+1-4 a^{3} p q+4 a^{2} q^{2}-4 a^{2} p^{2} q-4 a p a^{2}-2 a^{2} q-$ $4 a p-4 q<0, \Leftrightarrow\left(a^{2} q+a p q-2 a p-2 q+3\right)^{2}-8\left(a^{2} q+a p q-2 a p-2 q+3\right)+$ $8\left(a^{2} q^{2}-a p-q+2\right)<0$.

定理 2 对情形(C), 当 $a<b$ 时系统(1)存在极限环的充要条件是以下三条件同时被 满足: (i) $0<a<1, a b>1$; (ii) $0<a^{2}+a b-2<1-a^{2}$; (iii) $a^{4}+a^{3} b-a^{2}-2 a b+$ $\frac{1}{4}(a b+1)^{2}<0$.

证 因为文献[1]中定理 4 的条件(ii)、(iii)已满足,所以只需验证 (i). $M_{1}$ 是系统(1) 的稳定焦点,其等价条件是下面的三条:

(i) $\frac{1}{2}-2 \mu^{2}+2 \mu l-m>0, \Leftrightarrow a^{4} b^{2}-2 a^{3} b-a^{2} b^{2}+2 a b+a^{2}-1<0, \Leftrightarrow\left(a^{2}-\right.$ 1) $(a b-1)^{2}<0, \Leftrightarrow a^{2}<1 \Leftrightarrow 0<a<1(\because a>0)$;

(ii) $2 \mu l-2 m+\frac{3}{2}<0, \Leftrightarrow 2 a^{3} b+a^{2} b^{2}-4 a b-2 a^{2}+3<0, \Leftrightarrow(a b-1)$. $\left(2 a^{2}+a b-3\right)<0, \Leftrightarrow 2 a^{2}+a b-3<0(\because a b>1), \Leftrightarrow 0<a^{2}+a b-2<1-$ $a^{2}$. $\left(\because a^{2}+a b-2>0\right)$;

(iii) $4 \mu^{2} l^{2}+4 m^{2}+\frac{1}{4}-8 \mu l m-2 \mu l-2 m+8 \mu^{2}<0, \Leftrightarrow a^{6} b^{2}+a^{5} b^{3}-2 a^{5} b+$ $\frac{1}{4} a^{4} b^{4}+a^{4}-3 a^{4} b^{2}+3 a^{3} b-2 a^{3} b^{3}+\frac{7}{2} a^{2} b^{2}-a^{2}-2 a b+\frac{1}{4}<0, \Leftrightarrow \quad(a b-1)^{2}\left[a^{4}+\right.$ $\left.a^{3} b-a^{2}-2 a b+\frac{1}{4}(a b+1)^{2}\right]<0, \Leftrightarrow a^{4}+a^{3} b-a^{2}-2 a b+\frac{1}{4}(a b+1)^{2}<0$.

定理 3 对情形 (C), 当 $a>b$ 时系统(1)无极限环.

证 由 $M_{1}$ 在抛物线内部, 有 $\frac{1}{2}-2 \mu^{2}+2 \mu l-m>0$, 如定理 2 所证 $a^{2}-1<0$.

又由 $a>b$, 而 $\mu=\frac{a^{2} b}{2}>0$, 有 $b>0$, 所以有 $a>0$, 从而 $a^{2}>a b, 0>a^{2}-1>a b-$ 1.

于是 $\frac{\partial P}{\partial x}+\left.\frac{\partial Q}{\partial y}\right|_{M_{1}}=2 \mu l-2 m+\frac{3}{2}$

$$
-\frac{1}{2}(a b-1)\left[2\left(a^{2}-1\right)+(a b-1)\right]>0 .
$$

由此可知, 若 $M_{1}$ 为焦点, 则 $M_{1}$ 必为不稳定焦点, 根据文献 [1]的定理 1, 系统 (1) 此时无 1372 
极限环.

定理 4 对情形(D), 系统(1)存在极限环的充要条件是以下四个条件同时被满足:（i) $c>a b c>b>a>0$; (ii) $a b c(a+b+c)-2(a b+b c+a c)+3<0$; (iii) $[a b c(a+$ $b+c)-2(a b+b c+a c)+3]^{2}-8[a b c(a+b+c)-2(a b+b c+a c)+3]+8\left[a^{2} b^{2} c^{2}-\right.$ $(a b+b c+a c)+2]<0$; (iv) $a c+a b-2>0$.

证 $M_{1}$ 为系统(1)的稳定焦点,其等价条件为:

(i) $\frac{1}{2}-2 \mu^{2}+2 \mu l-m>0, \Leftrightarrow(a c-1)(a b-1)(b c-1)<0$. 由 $c>a b c>$ $b>a>0$ 可知上述不等式成立;

(ii) $2 \mu l-2 m+\frac{3}{2}<0, \Leftrightarrow a b c(a+b+c)-2(a b+b c+a c)+3<0$;

(iii) $4 \mu^{2} l^{2}+4 m^{2}+\frac{1}{4}-8 \mu l m-2 \mu l-2 m+8 \mu^{2}<0, \Leftrightarrow \frac{1}{4} a^{2} b^{2} c^{2}(a+b+c)^{2}+$ $(a b+b c+a c)^{2}+\frac{1}{4}-a b c(a+b+c)(a b+b c+a c)-\frac{1}{2} a b c(a+b+c)-(a b+$ $b c+a c)+2 a^{2} b^{2} c^{2}<0, \Leftrightarrow \quad[a b c(a+b+c)-2(a b+b c+a c)+3]^{2}-8[a b c(a+$ $b+c)-2(a b+b c+a c)+3]+8\left[a^{2} b^{2} c^{2}-(a b+b c+a c)+2\right]<0$.

此外, 文献 [1]定理 5 的条件(iii) 可以简化为:

$\frac{1}{2} b^{2}+b\left(\frac{a c}{2}-1\right) \cdot \frac{a+b+c}{2}+\left(\frac{a c}{2}-1\right)^{2}>0, \Leftrightarrow a^{2} b c+a b^{2} c+a b c^{2}-2 a b-$ $2 b c-4 a c+a^{2} c^{2}+4>0, \Leftrightarrow(a c-1+a b-1)[(a c-1)+(b c-1)]>0, \Leftrightarrow a c+$ $a b-2>0(\because a c>1, b c>1)$.

\section{参考文 献}

[1] 陈叔平,科学通报, 30(1985),6: 401-405. 